\title{
Proficient Masked Face Recognition Method Using Deep Learning Convolution Neural Network in Covid-19 Pandemic
}

\author{
Saeed A. Awan ${ }^{1}$, Syed Asif Ali², Imtiaz Hussain², Basit Hassan², Syed Muhammad Ashfaq Ashraf ${ }^{3}$ \\ ${ }^{1}$ DHA Suffa Univeristy, Ph-VII, DG-78, Khayaban-e-Tufail, Ext, Karachi, 75500, Pakistan \\ ${ }^{2}$ Sindh Madressatul Islam Univeristy, Aiwan-e-Tijarat Road, Karachi, 73000 \\ Pakistan \\ ${ }^{3}$ Muhammad Ali Jinnah University, Main Shahrah-e-Faisal, 22-E, Block-6, PECHS, Karachi-75400, \\ Pakistan
}

Received: June 5, 2021. Received: October 31, 2021. Accepted: November 24, 2021. Published: December 9, 2021.

\begin{abstract}
The COVID-19 pandemic is an incomparable disaster triggering massive fatalities and security glitches. Under the pressure of these black clouds public frequently wear masks as safeguard to their lives. Facial Recognition becomes a challenge because significant portion of human face is hidden behind mask. Primarily researchers focus to derive up with recommendations to tackle this problem through prompt and effective solution in this COVID-19 pandemic. This paper presents a trustworthy method to for the recognition of masked faces on un-occluded and deep learning-based features. The first stage is to capture the non-obstructed face region. Then we extract the most significant features from the attained regions (forehead and eye) through pre-trained deep learning CNN. Bag-ofword paradigm to has been applied to the feature maps to quantize them and to get a minor illustration comparing to the CNN's fully connected layer. In the end a Multilayer Perceptron has been used for classification. High recognition performance with significant accuracy is seen in experimental results.
\end{abstract}

Keywords- COVID-19, Face mask detection, Masked face detection, CNN, Deep learning, PCA, KNN, Neural Networks.

\section{INTRODUCTION}

$\mathrm{H}$ uman gathering, congested surfaces and polluted areas are the main sources of COVID-19 virus escalation. In order to maintain the social distance and keep untouched environment the conventional biometric systems which relies on fingerprints or passwords are not safe anymore. Current studies on COVID-19 virus have confirmed that wearing a face mask on public areas is playing a role of barrier between uninfected, healthy population and virus diffusion.
However, a masked face may cause the number of problems: (a) robbers and hoaxers may take mask advantage, robbery, stealing and committing crimes may carry out without any hassle. (b) with masked face, face authenticity and community access control mechanism have become very problematic. (c) current face recognition systems are insufficient to read face image description and recognize it. (d) in face recognition process revealing the nose region is significant which is the key point of pose correction, face normalization and face matching [1][2][3]. Therefore, masked face facing significant challenges in current face recognition methods.

In our work we distinguish face mask and masked face recognition is to tackle above problems. Face mask checks to a person with or without mask and applied it at public areas where mask is essential. Whereas the goal of masked face recognition to identify the subject's face wearing a mask depending on forehead regions, eyebrows, eyes and their distance.

In our paper, we focused on masked face recognition and proposing a deep learning approach to handle it. A pre-trained deep learning convolutional neural network (CNN) model has been used to capture the desired features from exposed facial regions in the image. In our case occlusions may occur in nose and mouth regions which has less hurdles to handle this problem proficiently.

The paper is organized as follows: A details review of available techniques and approaches is revealed in section 2 . In section 3 we discussed the face recognition system and proposed model. Experiments and results are presented in section 4 . We end the paper with conclusions and suggestions.

\section{LITERATURE REVIEW}

In contemporary COVID-19 pandemic everyone wears a mask to keep themselves safe from contracting virus infection. In this epidemic face recognition and identifications approaches have failed. It has created a massive dilemma for access 
control and authentication applications which depend heavily on face recognition.

Particularly, social security checks like bus stands, railway stations, shopping malls, airports and banks face recognition systems are failed to identify the masked faces. Whereas people will be on high risk without mask to pass authentication. Other security systems rely on touch (passwords or fingerprints) may also a cause of virus spreading.

With these barriers we need to improve existing face recognition methodologies that severely depend on facial feature points to perform individuality verification consistent in case of incomplete face pose. Masked face obstructs a majestic part of the face including the nose. To overcome on it multiple approaches have been proposed: local matching, discard occlusion and restoration approach [1].

A local matching approach requires the features be extracted from equal sized patches sampled from the image [22]. A matching process is then applied between the gallery and probe faces. A benefit of using this approach is that the patches do not overlap.

Another approach is recommended by [4] to identify people using partially exposed faces. To achieve this goal, they extracted geometrical and textural features and identified keypoints which are then used in point set matching. Using this method, the similarity between two faces is determined based on the distance between two sets of aligned features.

The Key-Points matching approach suggested by [5] uses a SIFT Key-Point Descriptor for selecting a key-points. These points are then used for recognition of partial face by applying Gabor Ternary Pattern and Point Set Matching.

Restoration approaches on the other hand attempt to restore the masked regions in the probe images according to the gallery images. [6] suggested restoration of facial occlusions by detecting occluded regions using threshold depth map values of a 3D image followed by Principal Component Analysis. Another prominent method in this approach is Statistical Shape Model suggested by [7] to predict and restore the partial facial curves. Iterative closest point algorithm also uses statistical estimation techniques to manage the occluded parts. Principal Component Analysis used to complete the partially visible curves.

Another set of techniques classified under the Occlusion Discarding, aim at excluding the occluded parts of the face. [8] used a Segmentation based method for firstly detecting and discarding the occluded and later using only the exposed parts. This was achieved by applying a SVM based classifier for detecting small local patches of masked portion of facial images whereas mean based weighted matrix used for unobstructed facial regions.

[9] used a mix of Discarding and Restoration approaches by making use of Global Masked Projection and Partial Gappy Principal Component Analysis with Eigen Vectors. Similarly, [10] experimented with Partial Matching Mechanism to eliminated masked regions and using unmasked regions for matching process.

It has become an established practice to use Convoluted Neural Networks for facial recognition problems when the face faces are hidden under masks [11][12].
Based on the human psychology factors, deep learning methods exploit the exposed parts of the face while completely ignoring the masked portions [13].

Tetrolet Local Directional Pattern Optimization [14] can also be used to extract facial features from the exposed sections of face which can then be used for classification purposes using Cat Swarm Optimization based 2-Dimensional Hidden Markov Model. This has resulted in high accuracy with reduced False Rejection Rate as well as False Acceptance Rate.

Another recent study [15] has made good use of Gabor Wavelet Feature Extraction Algorithm with Support Vector Machines for facial recognition.

\section{PROPOSED MODEL}

The masked face recognition process based on the gallery and the reference set. Gallery images are indexed on the bases of their resemblance to input query image as shown in figure 1.

Reference based descriptors are calculated through assessing resemblance between reference set and the gallery feature vectors for gallery images after pre-processing phase. The face recognition calculations need to manage huge measures of light varieties among display and test images. Every inquiry image is checked with gallery images, and the index calculation can be examined to aggregate match mark for credentials, and the receiver functional characteristic for authentication. From the gallery and query images noise is removed.

We extracted the Eigenfaces using PCA from the image dataset [16]. We calculated the covariance matrix of input image.

$C=\left(\begin{array}{ll}\sigma(x, x) & \sigma(x, y) \\ \sigma(y, x) & \sigma(y, y)\end{array}\right)$

After calculating the Eigenfaces each image is denoted as a weights' vectors. Finally incoming image's weights are compared with the database image's weights. Image is deliberated to be unknown when the weights of input image are greater than assigned threshold. And if weights are closest to the database image weights query image is identified.

\section{A. Local Bit Pattern (LBP)}

The LBP is a modest and very proficient texture operator allots a label to each pixel of an image by thresholding a $3 \times 3$ neighborhood with the center pixel value and considering the result as a binary number [17].

In this approach the decimal value which is greater or equal to threshold is replaced by binary 1 and others with 0 . Starting from top 8left neighbor, reading values clockwise the binary values have been attained which is revealed in figure 2 .

Euclidean distance between two histograms is calculated by following formula.

$D=\sqrt{\sum_{i=1}^{n}\left(\text { hist } 1_{i}-\text { hist } 2_{i}\right)}$

For pixel position (x,y), LBP is a systematic set of binary assessments of pixel strengths of central and its neighbor 
pixels. In [17][18] the 8-bits word is presented with decimal label values as follows:

$$
L B P(x, y)=\sum_{n=0}^{7} 2^{n} s\left(l_{n}(x, y)-l_{c}(x, y)\right)
$$

Where $l_{n}$ shows to grey values of the 8 neighbor pixels, $l_{c}$ to grey value of central pixel $(\mathrm{x}, \mathrm{y})$.

The function $\mathrm{s}(\mathrm{k})$ is given as:

$s(k)= \begin{cases}1 & \text { if } k \geq 0, \\ 0 & \text { if else }\end{cases}$

\section{B. K-Nearest Neighbour Classifier}

The K-Nearest Neighbour classifier purely depends on feature vectors distance. It categorizes mysterious data points by determining the most common class among the $\mathrm{K}$-closest examples.

As revealed in figure 3, the sample to be categorized signified by blue square. Which may be classified into green circles or orange triangles. When $\mathrm{k}=5$ orange triangles have $60 \%$ probability for classification which is higher than green circle $40 \%$. Blue square is classified into green circles having $60 \%$ probability which is greater than orange triangles $40 \%$ when $\mathrm{k}$ $=10$. We need to define Euclidean distance to apply K-NN classification.

$D(p, q)=\sqrt{\sum_{i}^{n}\left(q_{i}-p_{i}\right)^{2}}$

Where $q_{i}$ and $p_{i}$ are the values of the $i$ th variable at points $q$ and $p$ respectively and $n$ is the number of variables.

\section{Convolutional Neural Network}

Convolutional Neural Network (ConvNet) is a deep learning algorithm that is specialize for image recognition. It works in the same way as our visual cortex works and recognizes images. It gets an image as an input allocate learnable weights and biases to several phases in the image and be able to distinguish one from the other.

ConvNet architecture is divided into two broad Feature Learning and Classification sections. The input image enters into the extraction network, the extracted feature signals enter in the classification neural network which operates based on the feature of image and generates the output.

The ConvNet comprises of several layers revealed in figure 4 . A multi-dimensional array of numbers (image) is given to each layer as input and produces an output of a multidimensional array of numbers (image), which can be treated an input for the next layer. To classify images, an input image $\{64 \times 64\}$ is given to first layer and get a set of different categories $\{1 \times 1 \times 20$ categories in our case $\}$ as output of the final layer. One volume of activations to another via a differentiable function is transmuted at each layer. In our work we used three layers namely Convolutional Layer, Pooling Layer and Fully-Connected layer.

- INPUT Layer grips the raw pixel values of the image [64 $\times$ 64], we have image width 64 , height 64 in our data set.

- Convolutional layer generates feature maps from images, it does not employ connection weights and a weighted sum instead, and it contains convolutional filters that convert images. The filter of convolutional layers are two dimensional matrices having the result in volume such [64 $\times 64 \times 10$ ], with 10 filters.

- Component wise activation function RELU is applied, like $\max (0, y)$ with zero thresholding, due to which the size of the volume remained same $([64 \times 64 \times 10])$.

- Pooling layer reduced spatial size of the convolved feature shown in figure 5-a and 5-b, which decrease the computational power through down-sampling as result in volume $[32 \times 32 \times 10]$ and $[16 \times 16 \times 10]$ in dimensions (width, height) respectively. In our example, pooling layer produced output volume of size [112 x $112 \times 64]$ via pooled the input volume of size $[224 \times 224 \times 64]$ with stride 2 and filter size 2 . Since the volume deepness is conserved and the most communal reduce sampling operation is max, therefore each max is calculated over four numbers $\{2 \times 2$ square $\}$ [19].

- In Fully-Connected layer image is flatten into a column vector. At every iteration of training feed-forward and backpropagation is applied on flattened vector. After a number of epochs segregation is made between certain low level and dominating features by the model, resulting in volume size $[1 \times 1 \times 10]$.

The original image layer by layer transmuted from the original pixel values to the final class scores in ConvNet. The convolutional or fully connected layer do not only perform activation function in the input volume but also with weights and biases parameters as well. Moreover, a fixed function is implemented by POOL/RELU layers [20]. Gradient descent approach have been used to train the parameters in the convolutional/fully connected layer to keep class scores consistent that $\mathrm{CNN}$ computes for each image having labels in the training set [21].

In figure 6, to detect face layers and filters in a network are demonstrated. Initial edges are spotted at first layer, second layer detects features from preceding connected layers due to which it identifies more complex region of face like eyes, eyebrows, distance between eyes, dimension of foreheads, etc. Finally, the last layer detects the required face. 
INTERNATIONAL JOURNAL OF CIRCUITS, SYSTEMS AND SIGNAL PROCESSING

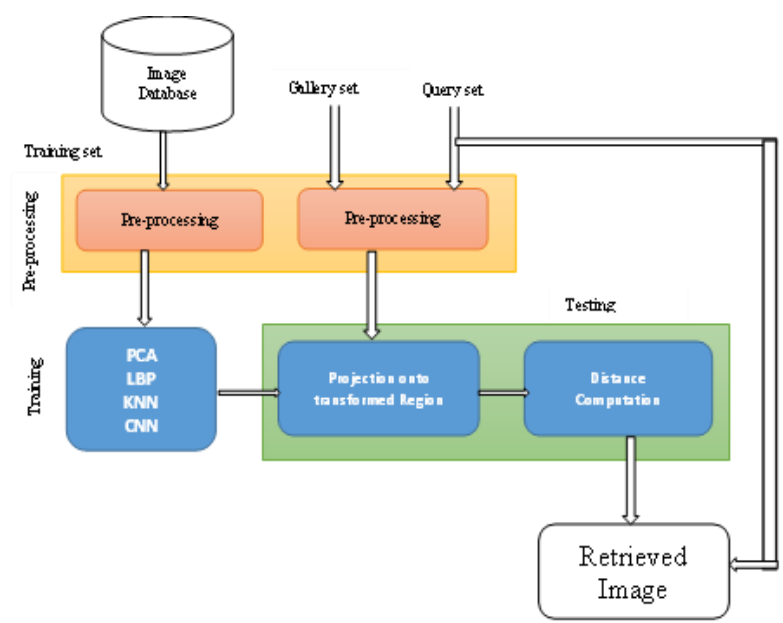

Figure 1. Masked Face Recognition Proposed Model

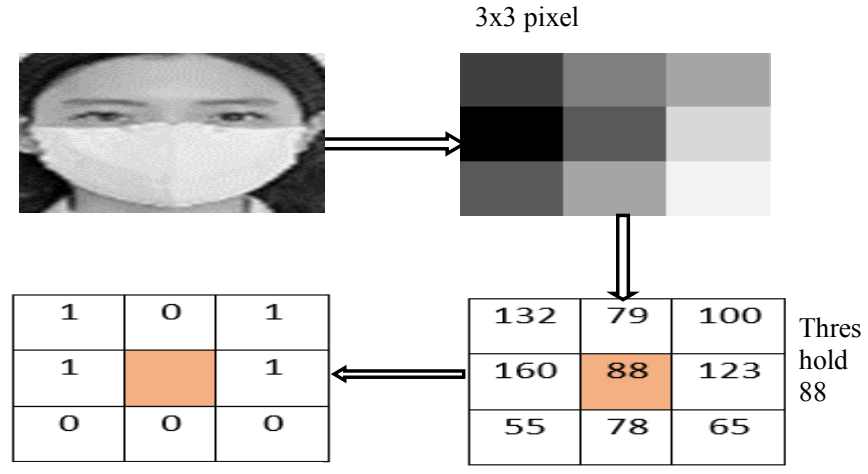

Binary 10111000

Figure 2 LBP Feature Extraction

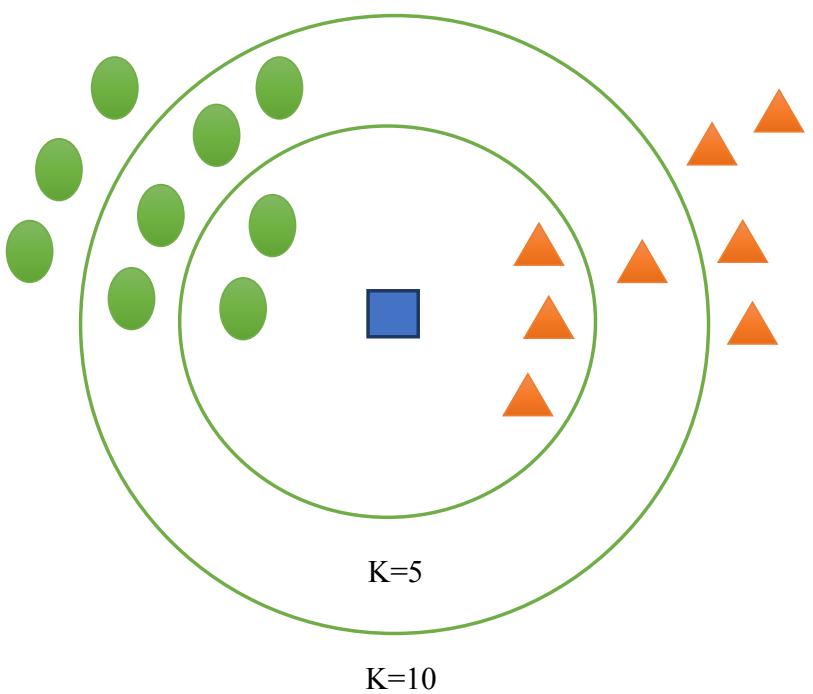

Figure 3. Principle Diagram of K-NN Classifier

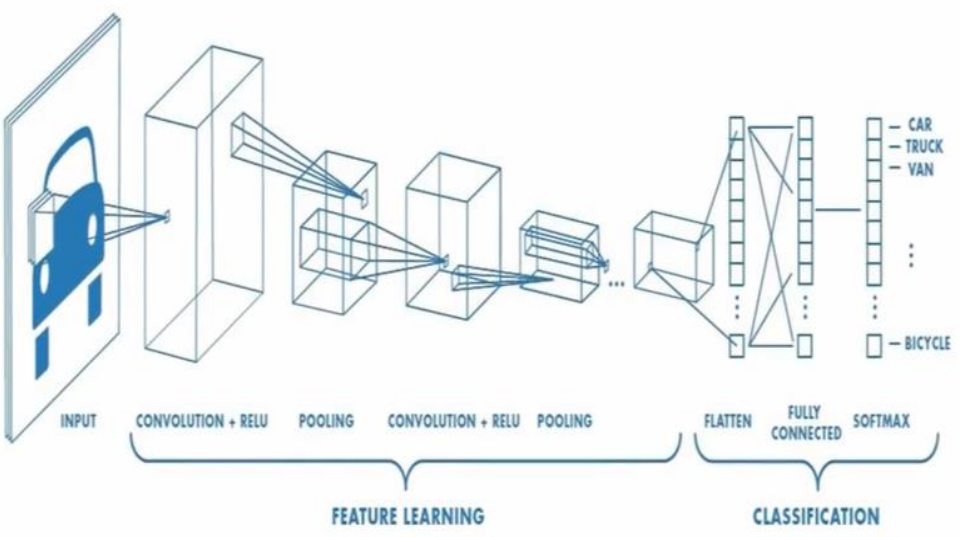

Figure 4. A Convolutional Neural Network Architecture [19]

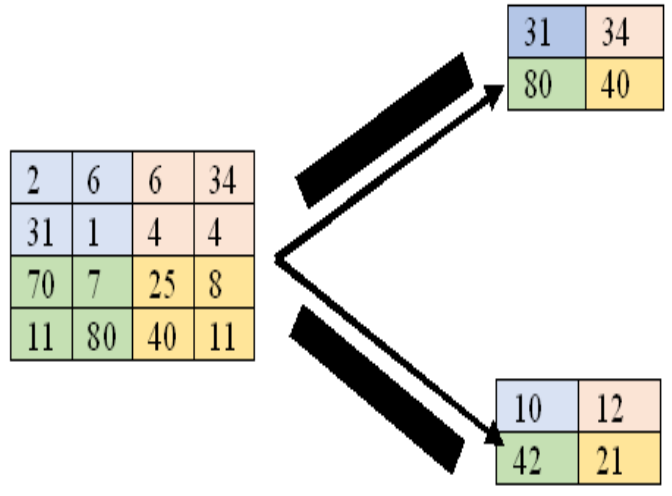

Figure 5a. Max Pooling Layer 
INTERNATIONAL JOURNAL OF CIRCUITS, SYSTEMS AND SIGNAL PROCESSING

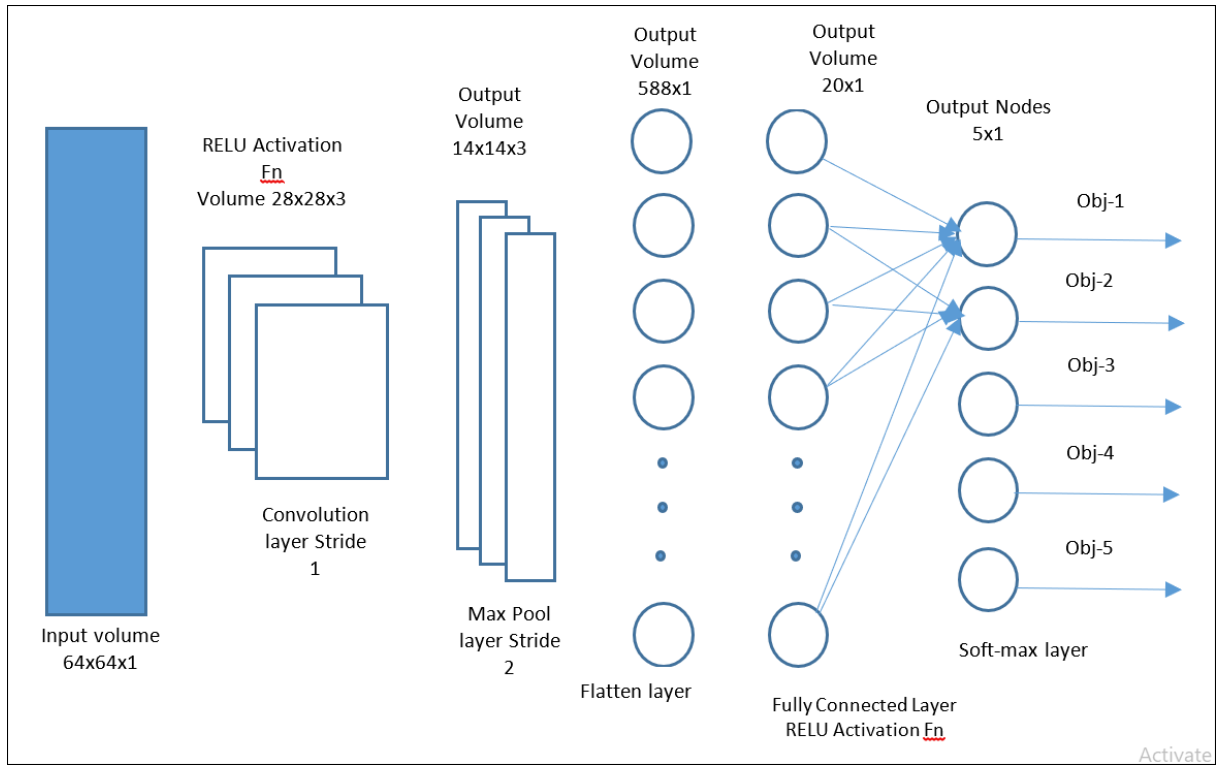

Figure 5b. Fully Connected Layer Classification

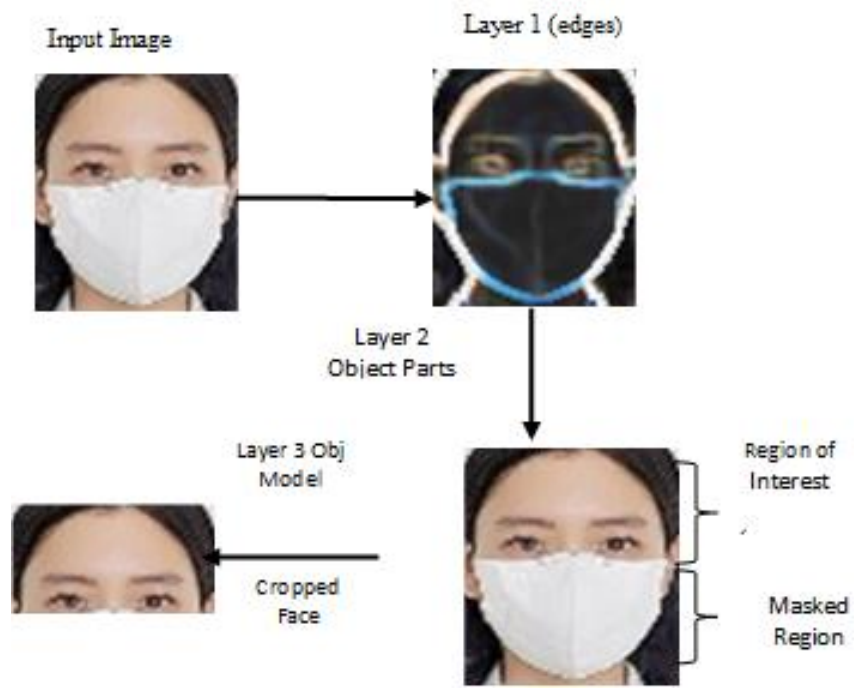

Figure 6. Sampling the asked face into regions

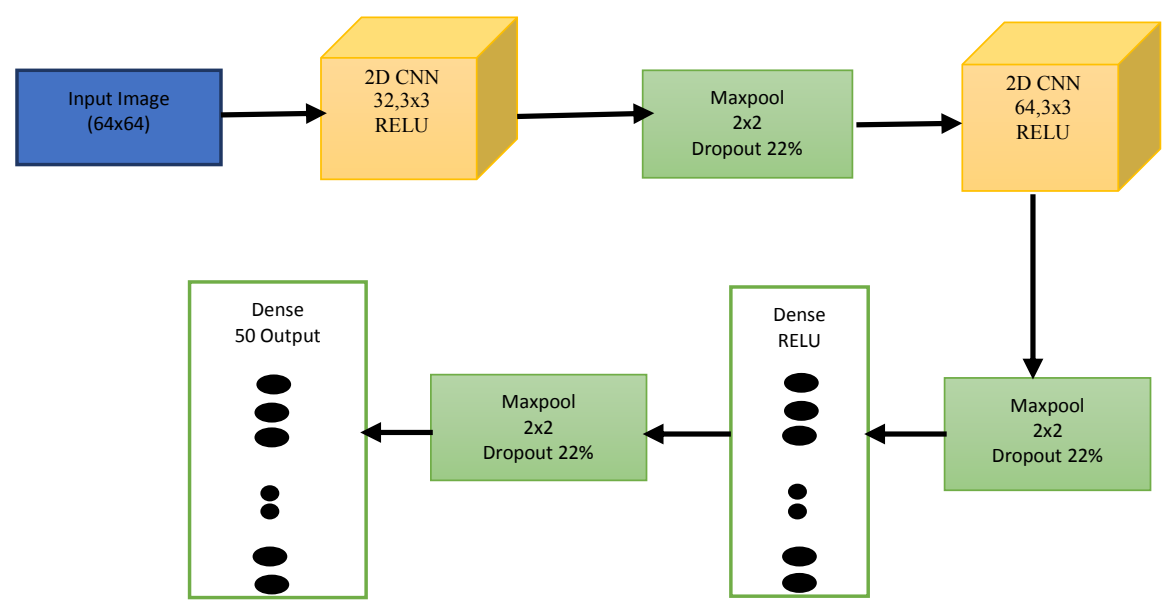

Figure 7. Block Diagram of Proposed ConvNet Method 


\section{EXPERIMENTS AND RESULTS}

On COVID-19 Kaggle masked face database we tested the execution of our proposed technique. We normalized and aligned our image dataset based on the location of human eyes. We used MATLAB and Python as a tool to implement all related methods in our work like LBP, PCA, KNN and pretrained CNN.

\section{A. Masked Face Dataset}

Our dataset is comprised of around 8000 images based on male, female, and children. This dataset has images with different modes such as medicated, non-medicated and ordinary mask, different styles of wearing mask and nonwearing masks as well and it also contains glasses and without glasses images. We used $80 \%$ to train our model and $20 \%$ to test the model from our dataset.

\section{B. Experiments}

Our proposed model follows the standard pattern to test all methods and approaches of the image recognition process presented in model diagram figure 1. Both images for training and testing are stored in the form of a vector. For description of feature vector the Euclidean distance was used.

We equated renowned algorithms PCA, LBP and KNN to estimate the efficiency of our proposed CNN method. After system training, Eignefaces are calculated by PCA, the indigenous features of object and its $2 \mathrm{D}$ texture analysis are mined through LBP. In our work, classification model is created using training dataset and its testing accuracy is evaluated by testing dataset through KNN classifier.

Table 1. The Convnet Layers Properties

\begin{tabular}{|c|c|c|}
\hline Layer No & Layer Type & Layer Attributes \\
\hline Layer-1 & Input & $64 \times 64$ \\
\hline Layer-2 & 1 Convolutional & $\begin{array}{lr}3 \times 3 & \text { karnel } \\
\text { dimension } & 32 \\
\text { feature maps } & \end{array}$ \\
\hline Layer-3 & Pooling & $\begin{array}{ll}2 \times 2 & \text { karnel } \\
\text { dimension } & \\
\text { Probability } & 22 \%\end{array}$ \\
\hline Layer-4 & 2 Convolutional & $\begin{array}{lr}3 \times 3 & \text { karnel } \\
\text { dimension } & 32 \\
\text { feature maps } & \end{array}$ \\
\hline Layer-5 & Pooling & $\begin{array}{ll}2 \times 2 & \text { karnel } \\
\text { dimension } & \\
\text { Probability } & 22 \%\end{array}$ \\
\hline Layer-6 & Fully connected-1 & 4000 neurons \\
\hline Layer-7 & Fully connected-2 & 50 neurons \\
\hline
\end{tabular}

According to above table, the original pixel values of an input image is altered to the final class values by forwarding it one by one layer.

An $\{64 \times 64\}$ image is given to input layer and 32 feature maps of $\{3 \times 3\}$ kernel dimension is extracted from it in $2 \mathrm{D} \mathrm{CNN}$ layer. RELU activations function was used due to which sparse features of the whole network were improved and neurons dependency was avoided for passing parameters. At MaxPooling layer $22 \%$ probability of output was dropped out by using $\{2 \times 2\}$ dimension kernel. The information and amount of data which needed to be processed on the upper level are provided by max-pooling method. Second 2D CNN used same parameters with double to 64 feature maps. With align of max-pooling standard dense layer having 4000 neurons and RELU activation function was used. After passing the last dropout output to softmax layer we got a classified distribution to 50 neurons and softmax activation function of final output. Training progress validation is achieved through softmax regression.

\section{Results}

This section presents acquired experimental results. The PCA's and LBP's algorithms recognition and overall accuracy is presented in the first and next two rows respectively. Third row presenting the KNN's algorithm accuracy, whereas the last row showing experimental results of proposed CNN.

Table 2. Face recognition accuracy for different Number of training images

\begin{tabular}{|c|c|c|c|c|c|}
\hline \multirow{2}{*}{ Methods } & \multicolumn{5}{|c|}{ Number of training and test images } \\
\cline { 2 - 6 } & $\begin{array}{c}\text { Training } \\
\text { Data \% }\end{array}$ & $1^{\text {st }}$ & $2^{\text {nd }}$ & $3^{\text {rd }}$ & $4^{\text {th }}$ \\
\hline PCA (\%) & 20 & 73.30 & 76.41 & 81.77 & 83.91 \\
\hline LBPH (\%) & 30 & 76.12 & 79.67 & 82.55 & 87.72 \\
\hline KNN (\%) & 50 & 70.63 & 74.80 & 78.30 & 82.10 \\
\hline $\begin{array}{c}\text { Proposed } \\
\text { ConvNet } \\
(\%)\end{array}$ & 80 & 92.66 & 95.01 & 97.00 & 98.86 \\
\hline
\end{tabular}

We divided all experimental results in to four groups. 1st $20 \%$ data for training (1600:8000), 2nd - 30\% data for training (2400:8000), 3rd - 50\% data for training (4000:8000) and 4 th $-80 \%$ data for training (6400:8000) is used. Testing was also performed for all groups of training data.

The outstanding results $(98.86 \%)$ were achieved by proposed ConvNet for 6400 trained images out of 8000 , whereas KNN algorithm gave worst results $(70.63 \%)$ for 4000 trained images out of 8000 .

Table 3. Comparison of different methods for acceptance, execution time, false rejection and false acceptance ratio of random images

\begin{tabular}{|c|c|c|c|c|l|}
\hline Methods & $\begin{array}{c}\text { Number } \\
\text { of } \\
\text { Images }\end{array}$ & $\begin{array}{c}\text { Accuracy } \\
\%\end{array}$ & $\begin{array}{c}\text { Time } \\
(\mathrm{s})\end{array}$ & FRR & FAR \\
\hline PCA & 400 & 83.91 & 76.41 & 0.126 & 0.003 \\
\hline LBPH & 1400 & 87.72 & 79.67 & 0.093 & 0.013 \\
\hline KNN & 6000 & 82.10 & 74.80 & 0.067 & 0.004 \\
\hline $\begin{array}{l}\text { Proposed } \\
\text { ConvNet }\end{array}$ & 7500 & 98.8 & 95.01 & 0.004 & 0.002 \\
\hline
\end{tabular}

Table 3 shows the comparison between proposed and other existing methods for acceptance ratio, execution time, false rejection ratio and false acceptance ratio values based on random number of images from database $\{400,1400,6000$, $7500\}$ respectively.

\section{CONCLUSION}

On ground reality human may use any object to occlude their faces in highly tensed (alive or death) scenarios. This creates 
hurdles and increase challenges for face recognition processes. Subsequently, present face recognition approaches will be insufficient to recognize occluded face. The generalization is improved for the face recognition system through proposed approach in the existence of mask layer on face. In order to accomplish this goal, we proposed a deep learning convolutional neural network method and quantization based approach to handle the recognition of faces with occlusion. We got high accuracy of recognition performance by our proposed approach. In our opinion, during the COVID-19 pandemic, this research work addresses the problem of occluded face recognition with efficient accuracy.

Video surveillance and violence video retrieval may handle by proposed method in future.

\section{REFERENCES}

[1] X. Peng, M. Bennamoun, A. S. Mian, "A Training-Free Nose Tip Detection Method from Face Range Images," Pattern Recognition, Volume 44, Issue 3, March 2011, Pages 544-558

[2] X. Lu, A. K. Jain, D. Colbry, "Matching 2.5D face scans to 3D models," IEEE Transactions on Pattern Analysis and Machine Intelligence, Volume 28, Issue 1, Jan. 2006

[3] M. L. Koudelka, M. W. Koch and T. D. Russ, "A Prescreener for 3D Face Recognition Using Radial Symmetry and the Hausdorff Fraction," 2005 IEEE Computer Society Conference on Computer Vision and Pattern Recognition (CVPR'05) - Workshops, 2005, pp. 168-168, doi: 10.1109/CVPR.2005.566.

[4] R. Weng, J. Lu and Y. Tan, "Robust Point Set Matching for Partial Face Recognition," in IEEE Transactions on Image Processing, vol. 25, no. 3, pp. 1163-1176, March 2016, doi: 10.1109/TIP.2016.2515987.

[5] Y. Duan, J. Lu, J. Feng and J. Zhou, "Topology Preserving Structural Matching for Automatic Partial Face Recognition," in IEEE Transactions on Information Forensics and Security, vol. 13, no. 7, pp. 1823-1837, July 2018, doi: 10.1109/TIFS.2018.2804919.

[6] P. Bagchi, D. Bhattacharjee, M. Nasipuri, "Robust 3D face recognition in presence of pose and partial occlusions or missing parts," International Journal in Foundations of Computer Science \& Technology (IJFCST), Vol.4, No.4, July 2014, arXiv:1408.3709

[7] Hassen Drira, Ben Amor Boulbaba, Srivastava Anuj, Mohamed Daoudi, Rim Slama. 3D Face Recognition Under Expressions, Occlusions and Pose Variations. IEEE Transactions on Pattern Analysis and Machine Intelligence, Institute of Electrical and Electronics Engineers, 2013, pp.2270 - 2283. ffhalshs-00783066

[8] G. N. Priya, W. Banu, "Occlusion invariant face recognition using mean based weight matrix and support vector machine," Sādhanā, Volume 39, issue 2, April 2014

[9] N. Alyuz, B. Gokberk and L. Akarun, "3-D Face Recognition Under Occlusion Using Masked Projection," in IEEE Transactions on Information Forensics and Security, vol. 8, no. 5, pp. 789-802, May 2013, doi: 10.1109/TIFS.2013.2256130,
[10]X. Yu, Y. Gao and J. Zhou, "3D face recognition under partial occlusions using radial strings," 2016 IEEE International Conference on Image Processing (ICIP), 2016, pp. 3016-3020, doi: 10.1109/ICIP.2016.7532913.

[11] A. Krizhevsky, I. Sutskever, G. E. Hinton, "ImageNet Classification with Deep Convolutional Neural Networks," Advances in Neural Information Processing Systems, 25(2), January 2012, DOI: 10.1145/3065386

[12] L. He, H. Li, Q. Zhang, Z. Sun, "Dynamic Feature Matching for Partial Face Recognition," IEEE Trans Image Process. 2018 Sep 18. doi: 10.1109/TIP.2018.2870946. Epub ahead of print. PMID: 30235130 .

[13]L. Song, D. Gong, Z. Li, C. Liu and W. Liu, "Occlusion Robust Face Recognition Based on Mask Learning With Pairwise Differential Siamese Network," 2019 IEEE/CVF International Conference on Computer Vision (ICCV), 2019, pp. 773-782, doi: 10.1109/ICCV.2019.00086.

[14]M. S. Meena, P. Singh, and A. Rana, "Tetrolet Local Directional Pattern and Optimization-Driven 2D-HMM for Face Recognition," Engineering World, Volume 1, Issue 2019, ISSN: 2692-5079, http://www.engw.org/articles/2019/9.pdf

[15] J. A. C. Moreano, N. B. L. S. Palomino, "Efficient Technique for Facial Recognition with Support Vector Machines in 2D Image with Cross-Validation in Matlab", WSEAS Transactions on Systems and Control, 15, 175183. https://doi.org/10.37394/23203.2020.15.18

[16] A. A. Aburomman and M. Bin Ibne Reaz, "Ensemble of binary SVM classifiers based on PCA and LDA feature extraction for intrusion detection," 2016 IEEE Advanced Information Management, Communicates, Electronic and Automation Control Conference (IMCEC), 2016, pp. 636640, doi: 10.1109/IMCEC.2016.7867287.

[17] J. Olivares-Mercado, K. Toscano-Medina, G. SanchezPerez, H. Perez-Meana and M. Nakano-Miyatake, "Face recognition system for smartphone based on LBP," 2017 5th International Workshop on Biometrics and Forensics (IWBF), 2017, pp. 1-6, doi: 10.1109/IWBF.2017.7935111.

[18] P. Kamencay, T. Trnovszky, M. Benco, R. Hudec, P. Sykora and A. Satnik, "Accurate wild animal recognition using PCA, LDA and LBPH," 2016 ELEKTRO, 2016, pp. 62-67, doi: 10.1109/ELEKTRO.2016.7512036.

[19]S. Saha, "A Comprehensive Guide to Convolutional Neural Networks," Towards Science, December 15, 2018, https://towardsdatascience.com/a-comprehensive-guideto-convolutional-neural-networks-the-eli5-way3bd2b1164a53

[20] L. Tobías, A. Ducournau, F. Rousseau, G. Mercier and R. Fablet, "Convolutional Neural Networks for object recognition on mobile devices: A case study," 2016 23rd International Conference on Pattern Recognition (ICPR), 2016, pp. 3530-3535, doi: 10.1109/ICPR.2016.7900181.

[21] S. Guo, S. Chen and Y. Li, "Face recognition based on convolutional neural network and support vector machine," 2016 IEEE International Conference on Information and Automation (ICIA), 2016, pp. 17871792, doi: 10.1109/ICInfA.2016.7832107. 
[22]A. M. Martinez, "Recognizing imprecisely localized, partially occluded, and expression variant faces from a single sample per class," in IEEE Transactions on Pattern Analysis and Machine Intelligence, vol. 24, no. 6, pp. 748-763, June 2002, doi: 10.1109/TPAMI.2002.1008382.

Saeed Ahmed obtained his Masters degree in Telecommunication offered in Department of Computer Sciences of IQRA University Karachi, Pakistan in 2008. Currently he is pursuing his Ph.D. in Artificial Intelligence. His research interest is the AI-based tools and techniques in Image processing particularly Steganography and Steganalysis. He is associated with the Department of Artificial Intelligence and Mathematical Sciences Sindh Madressatul Islam University Karachi.

Syed Asif Ali obtained his Ph.D. in Computer Science from University of Karachi in 2011. He has been involved in teaching and research for more than a decade. $\mathrm{He}$ is an approved Ph.D. supervisor of Computer Science and member of National Curriculum Revision Committee (NCRC) of Computer Science Subject by the Higher Education Commission (HEC), Govt. of Pakistan. He is a senior member of IACSIT, CSTA, ISTE and University Syndicate. He is an author of two books published by international Publisher LAMBERT, Germany.

Imtiaz Husain received his master's and a Ph.D. degree in Mathematical Sciences from the University of Karachi, Pakistan in 2008 and 2014 respectively. He is currently pursuing his Ph.D. in Artificial Intelligence. His research focuses on the application of AI-based tools and techniques in healthcare. He is an Assistant Professor in the Department of Artificial Intelligence and Mathematical Sciences Sindh Madressatul Islam University Karachi.

Basit Hassan holds a Master of Information Systems from the University of Melbourne, Australia, and a Master of Science in Computer Science from Iqra University, Karachi. He has been teaching at tertiary level a lecturer since 2012. Currently he is serving at Sindh Madressatul Islam University since 2015. Before that he served at private universities as full time as well as adjunct faculty. His experience also includes a brief two years at Algorithm Consulting (Pvt) Limited as a developer for AlgoERP an Enterprise Resource Planning for Apparel Industry.

Syed Muhammad Ashfaq Ashraf is currently serving in an R\&D organization (NESCOM). He received his Bachelor's degree in Electronics Engineering from NED-UET, Karachi in 2007 and pursuing MS in Computer Science from MAJU. He has over a decade experience of designing, implementation and management of wireless communication network. His research interests include Big Data Analytics, Automation and deep learning in healthcare.
Acronyms

\begin{tabular}{|l|l|}
\hline CNN & Convolutional Neural Network \\
\hline COVID-19 & Coronavirus Disease Of 2019 \\
\hline KNN & K Nearest Neighbor \\
\hline LBP & A.Local Bit Pattern \\
\hline PCA & Principal Component Analysis \\
\hline FRR & False Rejection Ratio \\
\hline FAR & False Acceptance Ratio \\
\hline
\end{tabular}

Creative Commons Attribution License 4.0 (Attribution 4.0 International, CC BY 4.0)

This article is published under the terms of the Creative Commons Attribution License 4.0

https://creativecommons.org/licenses/by/4.0/deed.en_US 